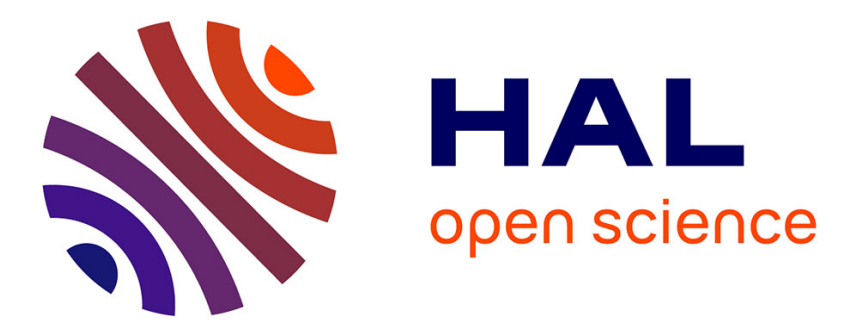

\title{
Les feuilles d'or de placage des casques celtiques d'Amfreville et d'Agris
}

Gérard Nicolini, Bernard Bouchet

\section{To cite this version:}

Gérard Nicolini, Bernard Bouchet. Les feuilles d'or de placage des casques celtiques d'Amfreville et d'Agris. Gallia - Fouilles et monuments archéologiques en France métropolitaine, 1990, 47, pp.67-77. 10.3406/galia.1990.2903 . hal-01916335

\section{HAL Id: hal-01916335 \\ https://hal.science/hal-01916335}

Submitted on 6 Feb 2020

HAL is a multi-disciplinary open access archive for the deposit and dissemination of scientific research documents, whether they are published or not. The documents may come from teaching and research institutions in France or abroad, or from public or private research centers.
L'archive ouverte pluridisciplinaire HAL, est destinée au dépôt et à la diffusion de documents scientifiques de niveau recherche, publiés ou non, émanant des établissements d'enseignement et de recherche français ou étrangers, des laboratoires publics ou privés.

\section{(ㅇ)(1) $\$$}

Distributed under a Creative Commons Attribution - NonCommercial - NoDerivatives $\mid 4.0$ 


\title{
LES FEUILLES D'OR DE PLACAGE DES CASQUES CELTIQUES D'AMFREVILLE ET D'AGRIS ${ }^{1}$
}

\author{
par Gérard NICOLINI et Bernard BOUCHET
}

Le microscope électronique à balayage $(\mathrm{MEB})$ permet de mieux percevoir les différentes méthodes de fabrication de la feuille d'or dans l'Antiquité. On peut ainsi distinguer une frappe primitive ou "verticale" et une frappe "progressive" plus élaborée. Les feuilles de placage des casques d'Amfreville et d'Agris sont issues de. la première méthode, pratiquement abandonnée dans le domaine méditerranéen à leur époque ( $\mathrm{VV}^{\prime \prime} \mathrm{s}$. avant $\mathrm{J}$.C.). Des observations au MEB plus nombreuses nous renseigneraient utilement sur l'extension du procédé dans le monde celte.

With the help of scanning electron microscopy (SEM) we are now able to distinguish between the various ancient methods of sheet gold hammering. A primitive or "vertical" hammering used from the beginning of gold jewellery has little by little been replaced by a more elaborate "progressive" hammering during the first millenium B.C. The plated gold leaves of the A mfreville and Agris helmets (4th century B.C.) were made with the first melhod which was virtually abandoned during the same period in the Mediterranean basin. Many other observations of this kind would be very useful to know the extension of the process in the Celtic world.

Après les récentes publications sur les casques d'Amfreville-sous-les-Monts (Eure) ${ }^{2}$ et d'Agris (Charente) $)^{3}$, consacrées à leurs aspects techniques, typologiques et stylistiques, il apparaissait intéressant de se tourner vers un problème non encore

1 Cette étude n'aurait pu être faite sans l'amabilité de Patrick Périn, Conservateur en chef des Musées de Rouen, et José Gomez de Soto, ER 27 du GNRS, inventeur du casque d'Agris, qui m'ont communiqué ces fragments, de Bernard Bouchet, responsable de l'examen des matériaux au MEB de l'École Nationale Supérieure de Mécanique et d'Aérotechnique de Poitiers. Qu'ils soient ici chaleureusement remerciés.

2 P.-M. Duval, Les Celtes, L'Univers des Formes, Paris 1977, p. 90-91, fig. 80 ; - V. Kruta, Etudes celtiques, XV, 2, 1978, p. 405-424; - A. Duval, J. Gomez de Soto, $1^{\text {er }}$ suppl. à Aquilania, 1986, p. 240-241; - A. Duval, L. Lғhoc7ky, V. SchaAf, Archäologisches Korrespondenzblatt 16, 1986, p. 8286 .

3 Parmi les publications déjà nombreuses, on retiendra abordé par les auteurs, celui de la fabrication de leurs feuilles d'or de placage. Celle-ci est désormais mieux perçue grâce à une série d'examens au microscope électronique d̀ balayage (MEB). Les modalités de la méthode sont décrites ci-après par $B$. Bouchet qui a largement contribué à l'adaptation du MEB à l'observation de la surface de la feuille d'or antique et moderne et à l'établissement d'une grammaire des signes selon les différents grandissements. On a pu ainsi reconnaître les signes de battage, de lissage et d'application, d'usure antique et moderne, d'attaque chimique des agents du sol de conservation des objets. Cette démarche a permis de

surtout : A. Duval, J. Gomez de Soto, op. cil., p. 241-244; J. Gомеz. DE Soто, Archäologisches Korrespondenzblatt, 16, 1986, p. 179-183, pl. V-VI; - C. EluèrF, J. Gomez DF Soto, A. R. Duval, Bulletin de la Société Préhistorique Française $(=B S P F), 84,198 \pi$, p. $8-22$ 
faire une comparaison entre les deux placages d'une part, entre ceux-ci et divers exemples de feuilles antiques et modernes, afin d'éviter certaines confusions, qui auraient évidemment remis en question le bien-fondé de la méthode. Afin que le lecteur perçoive bien tout ce qui a été vu par les auteurs, ceux-ci ont préféré appuyer leur démonstration sur un commentaire des photographies mêmes produites par le MEB.

\section{I.a feuille du Casque D'Amfreville}

Le fragment de feuille étudié appartient à la bordure supérieure du registre médian du décor du timbre. Rappelons que ce registre est formé d'une ligne de triscèles cantonnée de deux bandes ornées chacune d'une ligne de "cornes" dressées vers le centre, bordée du côté externe d'une ligne de bossettes masquant les têtes de clous. Le fragment mesure environ $32,5 \mathrm{~mm}$ sur $18 \mathrm{~mm}$; il comprend deux parties (fig. 1). La plus petite, à gauche, était écrasée. On y distingue cependant les traces de deux "cornes" et du trait limitant à l'intérieur la bande de bordure. Cette partie a été nettoyée par trempage dans l'eau distillée, sans frottement notable. La partie droile est plus importante; on y voit une "corne» et la ligne de bosseltes entre deux traits.

L'or du fragment paraît assez pur, de ton jaune à jaune clair. La partie droite a gardé une patine jaune verdâtre plus soutenue ${ }^{4}$. Aucune analyse n'en a été faite, mais le placage du casque d'Amfreville a été analysé par la technique de la microfluorescence $\mathrm{X}$ en plusieurs points et il n'y a guère de raison que ce fragment soit d'un or différent. Ces analyses ont produit: $\mathrm{Ag}: 1$ à $6 \%, \mathrm{Cu}: 0,6 \%{ }^{5}$. La couleur verdâtre de la surface pourrait être due à une altération de l'argent; elle a disparu lors du neltoyage à l'eau distillée ${ }^{6}$. On pourrait s'étonner de la faible teneur en cuivre car les feuilles de placage minces, notamment en bijouterie, ont souvent des teneurs supérieures qui assurent une bonne résistance à la

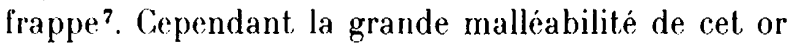

4 Sur la couleur et la patine des ors antiques, G. Nicolini, Techniques des ors antiques : la bijouterie ibérique

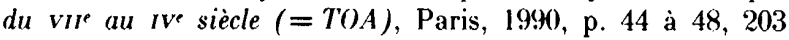
et passim.

5 C. Er.uére, J. (Gomez de Soto, $\Lambda$. R. DUVal, art. cit., p. 18.

6 Cif. note 4.

7 TOA, p. 37, 171-176. Par exemple, sur le placage de l'anneau de l'Acebuchal (or sur bronze) ibid., n' 2, p. 248, on trouve $\mathrm{Ag}: 30-35$, Cu: 6, A. Hartmann, Prähistorische Goldfunde aus Europa II, Studien zu den Anfängen der Metallurgie, Bd. 5, p. 114-115, Au 2077.

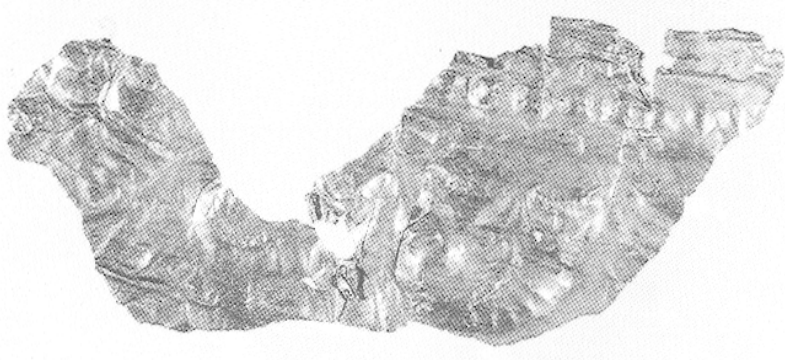

Fig. 1 - Casque d'Amfreville. Fragment étudié de la feuille de placage.

facilitait sans doute le brunissage sur le support. En outre si l'on excepte le casque d'Agris (infra), cetle composition est fréquente dans les ors gaulois, employés en placage ou massifs. Il pourrait s'agir d'ors naturels non alliés ${ }^{8}$.

I'épaisseur de la feuille est irrégulière, variant de 3 à $4 / 100^{\circ}$ environ, selon les mesures au palmer. Il est difficile de dire aujourd'hui si ces irrégularités sont dues entièrement à la technique de frappe (v. infra) ou au brunissage, ou aux deux à la fois. Quoi qu'il en soit, cette épaisseur est importante pour un placage si l'on considère les placages d'or sur bronze en bijouterie, généralement beaucoup plus minces ${ }^{9}$. Là, l'épaisseur de la feuille peut avoir plusieurs raisons : le désir de donner une plus grande valeur à la pièce, une certaine impéritie dans la technique du placage, une trop faible résistance de l'or, peut-être les trois à la fois. La technique adoptée ètait certainement le brunissage sur le support travaillé au repoussé, dont le relief et le décor apparaissaient ainsi sur la feuille d'or, dans un procédé s'apparentant à celui du travail sur la forme en relief (Modellieren des Allemands) ${ }^{\mathbf{1 0}}$. La feuille était ensuite détachée puis encollée sur l'envers et de nouveau mise en place. Il était en effet impossible de faire tenir une feuille aussi épaisse sans la coller. Il serait d'ailleurs intéressant d'analyser les restes de matière organique (?) sur le revers.

La méthode de frappe et le lissage de la feuille d'une part, le brunissage pour l'application de celleci sur le support de bronze d'autre part, apparaissent sur les macrophotographies optiques reproduites ici, issues de clichés $\times 3$ à $\times 9$ sur l'émulsion. Sur l'avers, on distingue autour des bossettes (fig. 2) des

8 Cf. note 5 sur les ors naturels, $T O A$, p. 12-14. Il est dommage que nous ne disposions pas d'analyses des traces pour les deux casques.

9 Cf. note 6 et $T O A$, p. 60 et 75 .

10 Ibid., p. 76 et 81-82. 


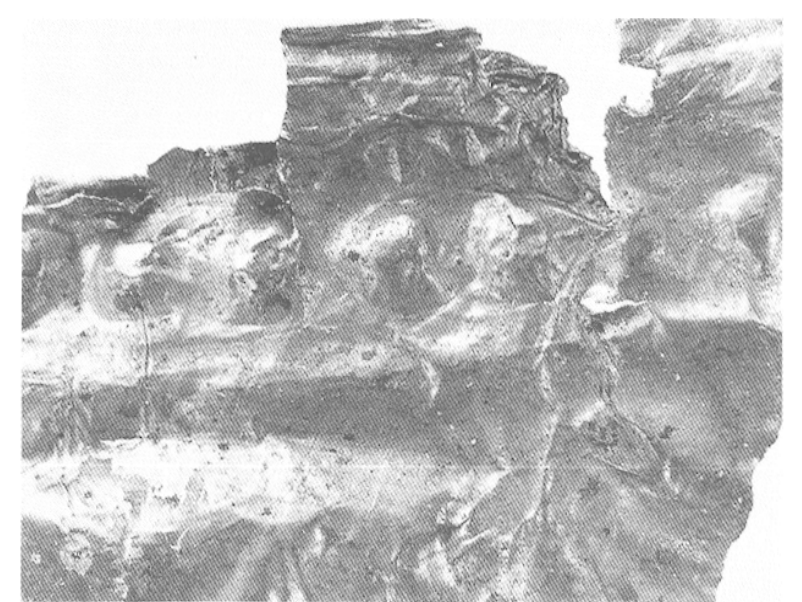

Fig. 2 - Casque d'Amfreville, avers de la feuille de placage. Impacts de battage.

impacts de battage caractérisés par des anfractuosités irrégulières, en partie comblées par le lissage, remplies d'une matière rougeâtre, peut-être identifiable à la patine rouge fréquente sur les bijoux ${ }^{11}$. Le brunissage sur le support à reliefs a produit des gaufrures caractéristiques du procédé, en particulier sur la ligne en relief entre les bossettes et les "cornes». Il est surtout visible sur les parties plates autour des reliefs (fig. 3), au bord inférieur du fragment, où il a laissé des stries peu ordonnées. Les gaufrures sont visibles aussi dans cette partie, de même que les impacts de frappe (anfractuosités remplies de noir) sur les reliefs de la "corne». Enfin, sur la "corne» elle-même (fig. 4), le brunissage n'est pas perceptible, masqué probablement par l'usure, mais il a néanmoins provoqué une gaufrure longitudinale et une perforation sur le sommet du relief. Sur le revers (fig. 5), les gaufrures sont également présentes, mais non écrasées par l'outil comme sur l'avers. Le semis de petites dépressions a sans doute été provoqué par le grain de la plaque de bronze. On aperçoit aussi des restes de matière brunâtre déjà signalés plus haut, qui pourraient être de la colle ou un agent collant organique. L'apport des macrophotographies n'est donc pas négligeable. Cependant les photos au MEB permettent de préciser la méthode de frappe utilisée. Elles concernent toute la petite partie du fragment (côté gauche) qui a subi le nettoyage sommaire mentionné plus haut, effectué en vue d'une meilleure observation. On verra qu'il n'a pas éliminé totalement les impuretés. Les photographies sont présentées dans un ordre croissant de grandissement.

11 Cf. note 4

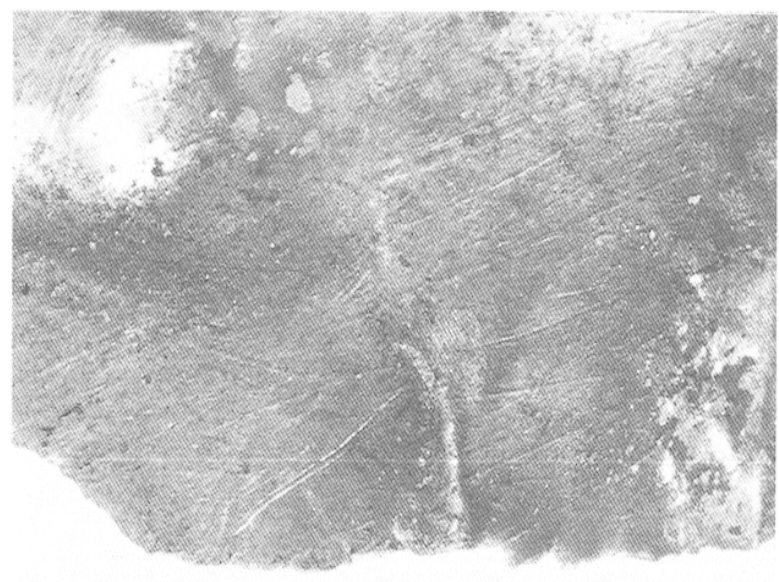

Fig. 3 - Casque d'Amfreville (avers). Gaufrures caractéristiques du brunissage.

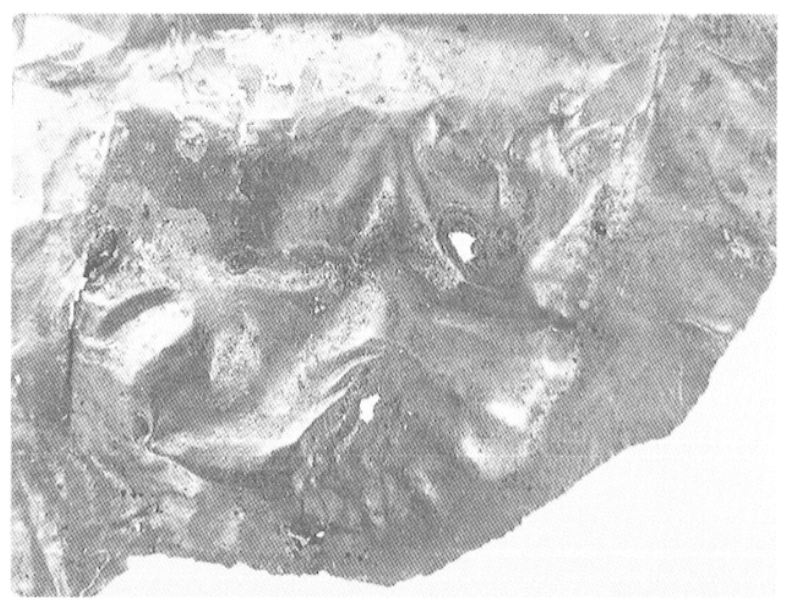

Fig. 4 - Casque d'Amfreville (avers).

Gaufrure et perforation provoquées par le brunissage.

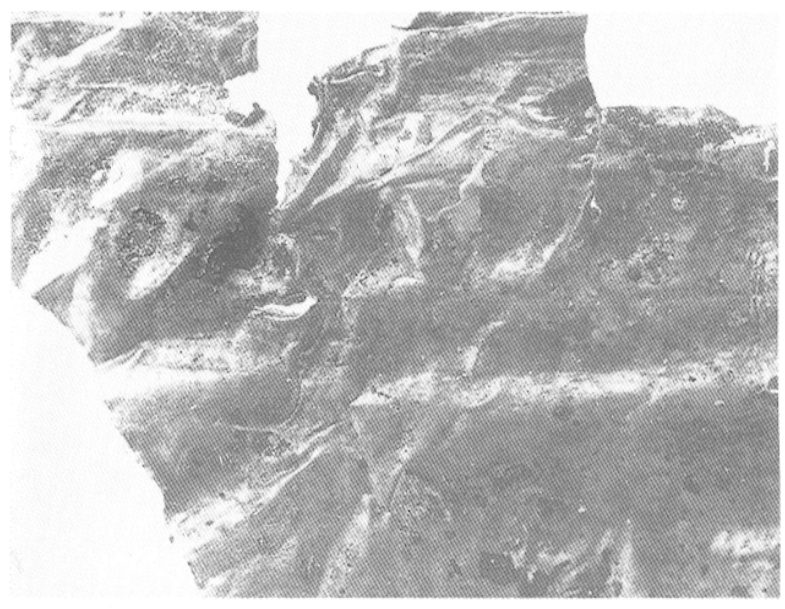

Fig. 5 - Casque d'Amfreville, revers de la feuille de placage. Gaufrures non écrasées. 


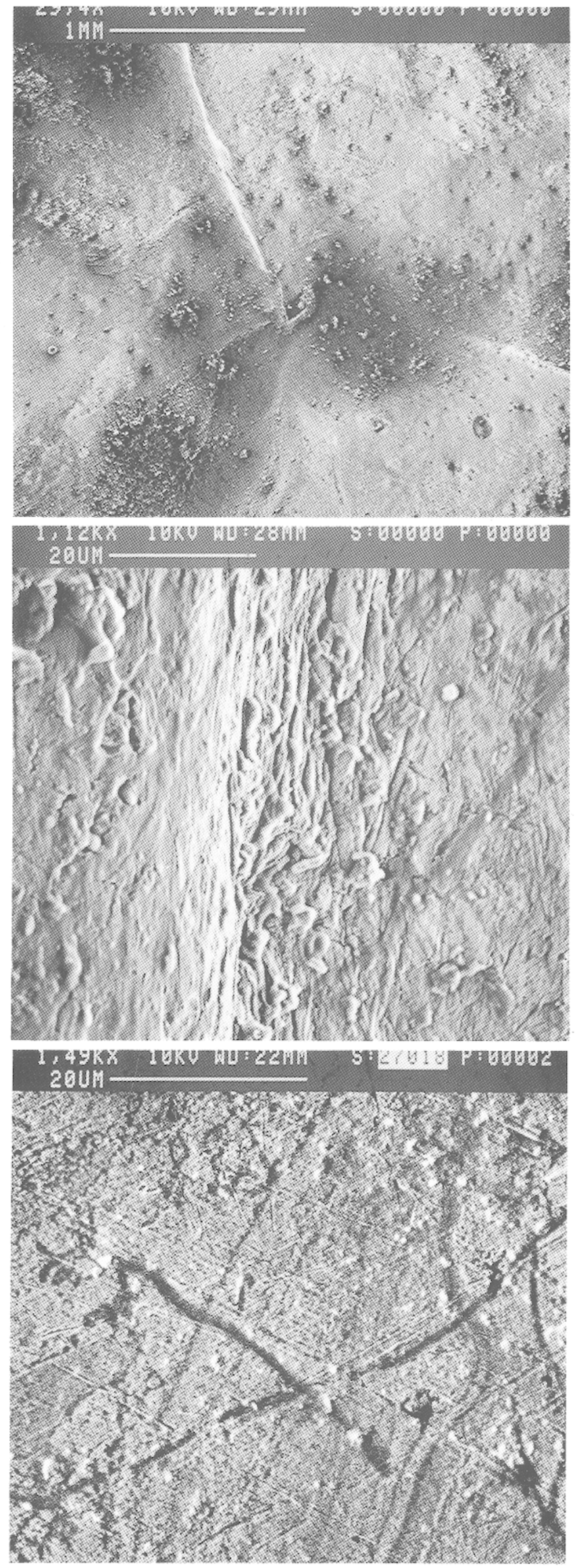

\section{Petite partie dU fragment, prés du CENTRE}

A ce modeste grandissement, la surface paraît relativement lisse, parsemée d'impuretés très adhérentes, généralement minérales (haute teneur en $\mathrm{Ca}$ décelée à la microsonde) souvent logées dans les anfractuosités produites par le battage ou des impacts postérieurs à la fabrication. Une grande concentration d'impuretés provoque un assombrissement sans rapport avec la couleur du métal. Les deux lignes claires qui rayonnent de la lacune du centre sont des gaufrures provoquées par le brunissage sur le support de bronze (v. supra). Elles se présentent comme des escarpements regardant vers la droite et vers le haut. La lacune centrale est un arrachement de la surface, également provoqué par le brunissage.

Fig. 6 - Casque d'Amfreville. Photo MED ENSMA $\times 29,4,10 \mathrm{KV}$, WD $29 \mathrm{~mm}$ (27 janvier 1988).

\section{DÉTAIL DE LA FIGURE 6}

On aperçoit dans la partie gauche le front de la gaufrure de brunissage, orienté du haut vers le bas, regardant vers la droite, au bas duquel la poussèe a fait saillir le métal en petits bourrelets parallèles. Dans la partie basse, à droite, non recouverte par le déferlement du métal, on distingue la surface de la feuille hors de l'atteinte du brunissoir. Elle présente de petites rayures de lissage et, dans le coin inférieur droit, une "nappe» écrasée avec un lobe saillant vers le bas. C'est une trace de battage de la feuille, au même titre que la large ligne sinueuse foncée qui disparaît en bas sous le front de la gaufrure (v. infra). Nombreuses impuretés de teinte claire.

Fig. 7 - Casque d'Amfreville. Photo MEB EnSMA $\times 1120,10 \mathrm{KV}, \mathrm{WD} 28 \mathrm{~mm}$ (27 janvier 1988).

\section{Petite PARTIE DU FRAGMENT, PRĖS DU CENTRE}

La surface est parsemée de nombreux accidents de faible amplitude. On distingue d'abord des sillons sinueux sombres entrecroisés et assez désordonnés qui sont en réalité les racines des nappes successives produites lors du battage (v. infra). Beaucoup moins importantes sont les stries de lissage, rectilignes et parallèles, qui traversent obliquement l'image, principalement de l'angle en bas à gauche à l'angle en haut à droite mais qui sont perceptibles sur tout le champ. Quelques stries perpendiculaires apparaissent faiblement vers le centre. Les dépressions très irrégulières sont provoquées par l'attaque chimique des agents du sol. Impuretés claires.

Fig. 8 - Casque d'Amfreville. Photo MEB ENSMA $\times 1490,10 \mathrm{KV}$, WD $22 \mathrm{~mm}$ (27 janvier 1988). 

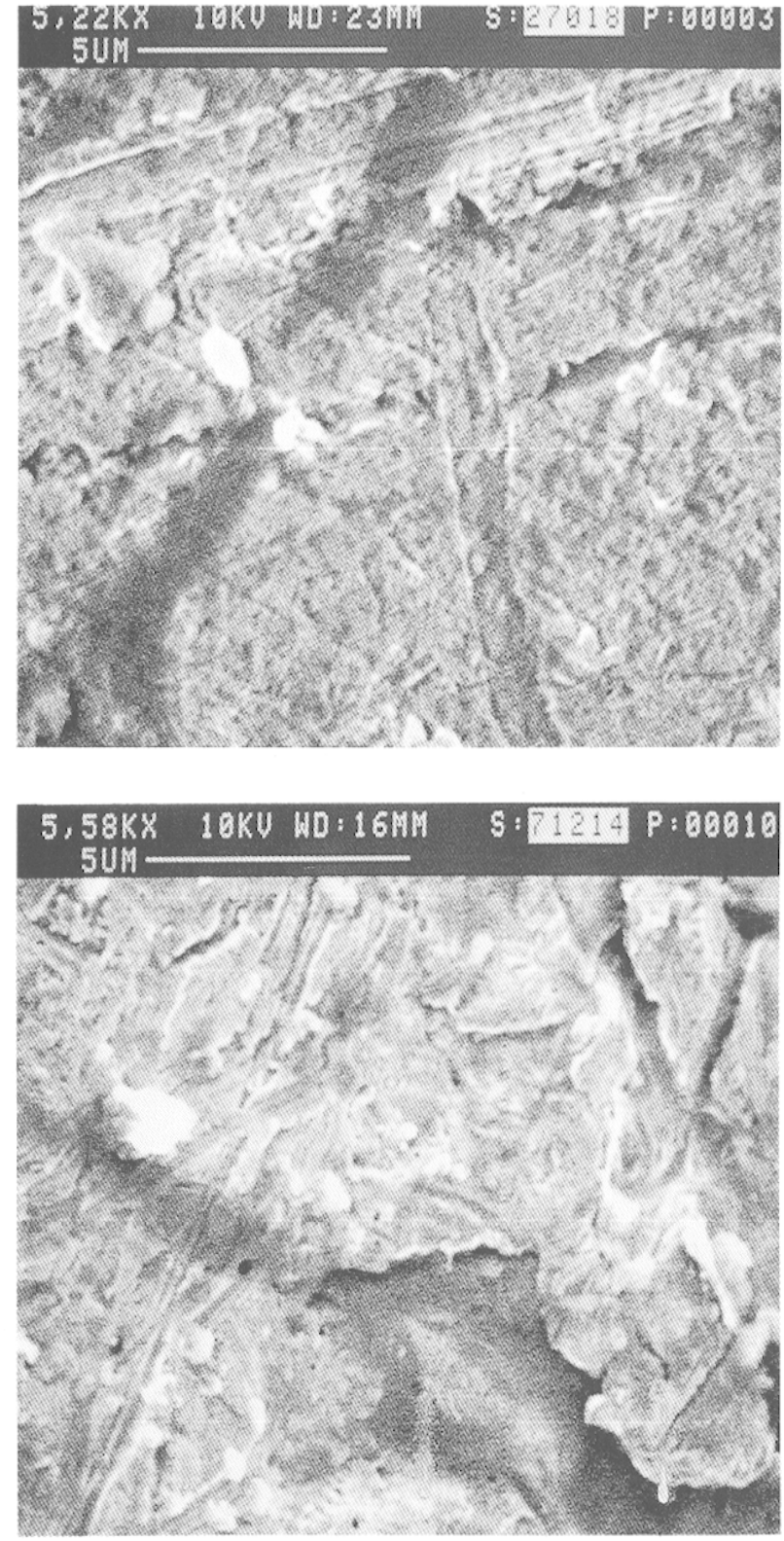

\section{DÉTAIL DE LA FIGURE 8}

A ce grandissement important, on aperçoit une large racine de nappe, de l'angle inférieur gauche au centre en haut, recoupant obliquement deux autres racines parallèles, elles-mêmes recoupées par un accident dû au lissage. Celui-ci apparaît surtout sous la forme de stries obliques dans la partie supérieure de l'image, recoupant les racines de nappe. Attaque chimique modérée. Quelques stries d'usure antique. Grosses impuretés à gauche.

Fig. 9 - Casque d'Amfreville. Photo MEB E.NSMA $\times 5220,10 \mathrm{KV}$, WD $23 \mathrm{~mm}$ (27 janvier 1988).

\section{Petite PaRtie dU FRAGMENT, PRÉS DU BORD GAUCHE}

L'image présente une importante racine de nappe dans la moitié inférieure, élargie vers le bas et encore partiellement recouverte par le métal de la nappe elle-même qui a jailli du haut vers le bas. Les "canaux» en $Y$ de la partie droite sont également dus à la frappe et recreusés par l'attaque chimique probablement. Les stries perpendiculaires à la racine de la nappe sont dues au lissage. Impuretés claires.

Fig. 10 - Casque d'Amfreville. Photo MEB ENSMA $\times 5580,10 \mathrm{KV}$, WD $16 \mathrm{~mm}$ (14 décembre 1987).

\section{LA FRAPPE PRIMITIVE ET LA FEUILLE DE PLACAGE}

Les macrophotographies (fig. 2 à 5 ) et plus encore les photos au MEB (fig. 6 à 10) permettent d'attribuer la fabrication de la feuille de placage du casque d'Amfreville à ce que l'on peut appeler la frappe verticale ou primitive, qui se développe depuis les origines de la métallurgie de l'or au $\mathrm{V}^{\mathrm{e}}$ millénaire puis décline au début du $\mathrm{I}^{\mathrm{er}}$ millénaire où elle est progressivement abandonnée. On la retrouve cependant çà et là, notamment dans les régions périphériques, comme en Ibérie et en Gau- le ${ }^{12}$. Sans en rappeler le détail, on peut dire qu'elle consiste grosso modo à frapper verticalement la pépite ou le lingot à intervalle plus ou moins régulier, ce qui provoque une alternance de dépres-

12 TOA, p. 76-80, pl. 4 à 6,217 a-d. On verra notamment $\mathrm{pl}$. $6 \mathrm{~d}$ une photo $\mathrm{MEB} \times 500$ d'une lamelle d'or ibérique de Castellar (Jaén) datée du Iv"s., donc à peu près contemporaine des casques d'Amfreville et d'Agris, qui présente la mème technique de frappe primitive. 


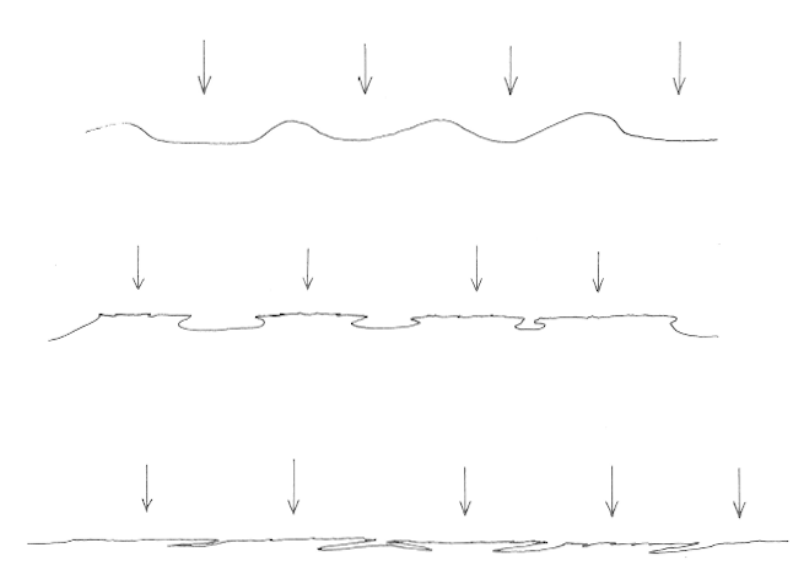

Fig. 11 - Les étapes de la frappe verticale et le lissage de la feuille. sions et de reliefs. Lorsqu'on frappe ensuite sur les reliefs vigoureusement pour niveler la surface, le métal s'échappe latéralement sous forme de vaguelettes, puis de véritables "nappes" qui évoquent les nappes de charriage de la tectonique. Le batteur cherche ensuite à faire disparaître ces nappes en poursuivant l'écrasement vertical, ce qui peut provoquer d'autres nappes, ou en lissant la surface de manière à écrêter les nappes. Il reste alors à la surface un sillon ou racine de nappe, résultant du repli initial du métal. Un lissage ou un polissage très accusé peut effacer presque totalement ce sillon (fig. 11). Lorsque la frappe est reprise en tous sens, les sillons sont naturellement entrecroisés (fig. 8 à 10). Cet enchevêtrement caractérise généralement la frappe primitive. On notera qu'il apparaît sur la feuille d'Amfreville à l'aide des grandissements MEB supérieur's à $\times 1000$, le signe étant imperceptible sur les vues $\times 100$ environ. J'ai tenté de reproduire l'effet en atelier. L'expérience s'est soldée par un demi-échec car il est impossible encore, et nous devons nous en féliciter, de reproduire l'aspect antique d'une feuille battue selon ce procédé : on ne peut reconstituer l'usure antique et l'attaque chimique du sol et surtout le lissage primitif. Ces imitations ne résistent pas à l'examen au MEB, comme on le voit sur les figures 12 et 13 .

Plaque de 1/10: D'or vert (Au $A G, C U=0$ )

Début de frappe primitive verticale. L'or vert a été choisi pour son absence de cuivre et une meilleure réponse au MEB. Malgré le fort grandissement, on aperçoit distinctement le début de la formation des vaguelettes dont le sens de progression est généralement vers le bas de la vue. Nombreux impacts de corps étrangers de 1 à 2 microns $(0,002 \mathrm{~mm})$ de largeur pour la plupart.

Fig. 12 - Plaque d'or vert. Photo MEB ENSMA $\times 1500,15 \mathrm{KV}$, WD $20 \mathrm{~mm}$ (12 avril 1988). 


\section{FEUILLE D'OR VERT}

Frappe primitive verticale en cours, suivie d'un lissage au maillet. La frappe a été exécutée au maillet de chène de Provence. La feuille a atteint $6 / 100^{\mathrm{e}}=60$ microns. Le bord de la feuille est normalement déchiqueté. Les trois quarts de la vue sont occupés par une avancée du métal qui tend à recouvrir une autre région de la feuille dans l'angle inférieur droit. Le lissage a décapité plusieurs nappes dans la partie inférieure gauche de la vue. Il en subsiste les sillons très resserrés, de direction gauchedroite. Le plus important de ces sillons de racine de nappe s'élargit en une anfractuosité triangulaire (fig. 9). D'autres nappes sectionnées en arc de cercle sont visibles au centre et à gauche, le sillon de l'une rejoignant presque l'anfractuosité. Le lissage au maillet effectué en mouvement circulaire a laissé des stries peu marquées. Les impacts de frappe ont presque disparu de ce fait.

\section{La feuille du CASQue D'Agris}

Le fragment étudié, de très modeste dimension, 9 sur $13 \mathrm{~mm}$ environ, n'a pu être situé sur le casque avec précision. A l'œil nu, c'est un or jaune mat, qui semble dépourvu de patine, mais non exempt de taches (fig. 14). De nombreuses analyses ont été pratiquées sur les feuilles de placage du casque d'Agris et, là encore, la composition du fragment ne doit pas être différente. Il s'agit d'un or affiné qui comprend en moyenne : $\mathrm{Au}: 98$ à $99 \%, \mathrm{Ag}: 0,5 \%$, $\mathrm{Cu}: 0,2 \%^{13}$. On comprend mal, à vrai dire, les raisons de l'utilisation d'un or affiné dans une feuille de placage. Sa faible teneur en cuivre lui donnait, il est vrai, une plus grande malléabilité comme dans le cas du casque d'Amfreville. Néanmoins, lorsque l'on place l'un à côté de l'autre les fragments des deux casques, celui d'Agris paraît. plus jaune et plus éclatant. Il est donc probable que le choix d'un or affiné est la preuve d'une recherche d'effet, d'une délicatesse d'exécution supérieure encore à celle de l'orfèvre d'Amfreville.

L'épaisseur varie de 4 à $5 / 100^{\circ}$ et semble supérieure à celle du fragment d'Amfreville, autant que l'on puisse en juger sur un si petit espace, mais elle est encore supérieure en d'autres endroits ${ }^{\mathbf{1 4}}$. Cette irrégularité est donc commune aux deux casques, pour lesquels on a usé du même procédé d'application (v. supra). Les macrophotographies ne révèlent pas de très grandes différences dans la

13 Cf. note 5.

14 C. Eluère a mesuré 70 microns ailleurs, mais ne précise pas l'endroit, $B S P F, 84,1987$, p. 10.

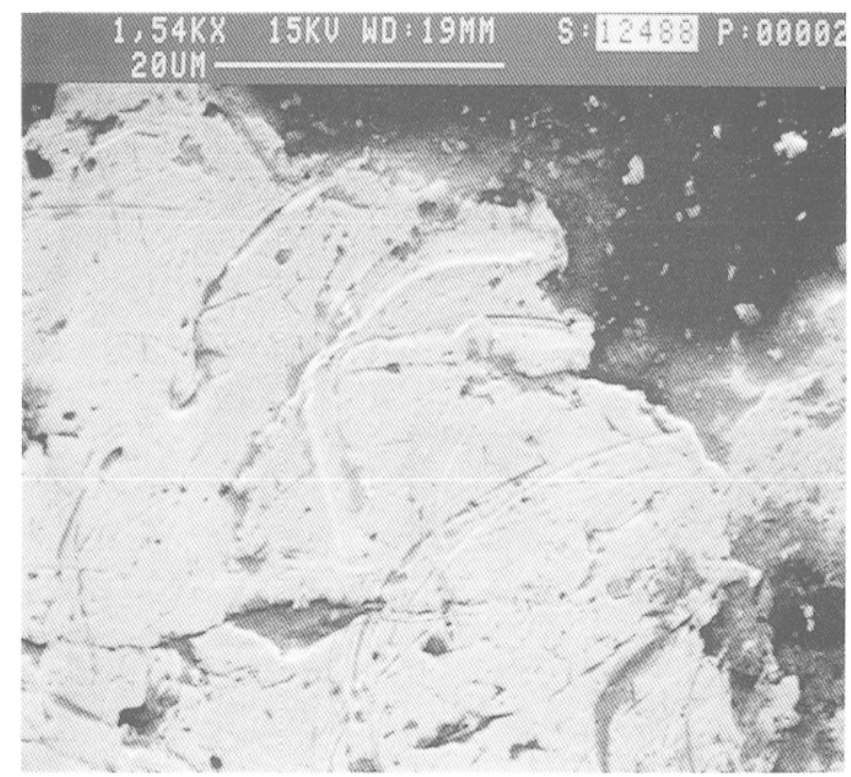

Fig. 13 - Feuille d'or vert. Photo MEB E.NSMA $\times 1540,15 \mathrm{KV}$, WD) $19 \mathrm{~mm}$ (12 avril 1988).

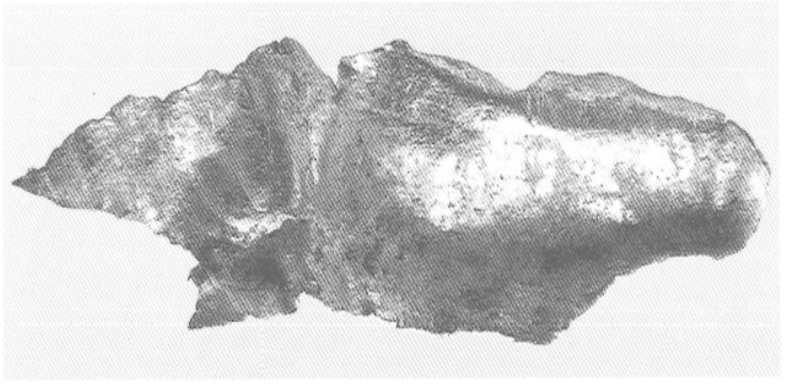

Fig. 14 - Casque d'Agris. Fragment de la feuille de placage.

méthode de frappe et le brunissage. On trouve les mêmes impacts, gaufrures, lacunes, vaguelettes et nappes (fig. 15 et 16). Cependant quelques zones granuleuses ont peut-être été provoquées par l'emploi d'une peau entre le marteau et l'enclume ou le tas. Cette Lederstruklur ${ }^{15}$, si elle existe bien ailleurs, détail qui mériterait d'être vérifié, serait l'indice d'une qualité supérieure du travail, mais le fragment est trop modeste pour qu'on puisse affirmer que le grain est bien celui d'une peau. Ce sont les photographies au MEB qui vont permettre de dégager les caractéristiques de la fabrication de la feuille d'Agris. La face A correspond au revers de la feuille (face en contact avec la plaque de bronze), la face B à l'avers (face visible). Il a paru préférable d'observer d'abord la face A (revers), exempte de traces d'usure moderne.

15 Sur la Ledersiruktur détectee par V. von Claer, voir H. Hoffmann, V. von Ci.afr, Antiker Gold und Silberschmuck, Mayence, 1968, p. 195-197, fig. 131 b;-TOA, p. 71. 


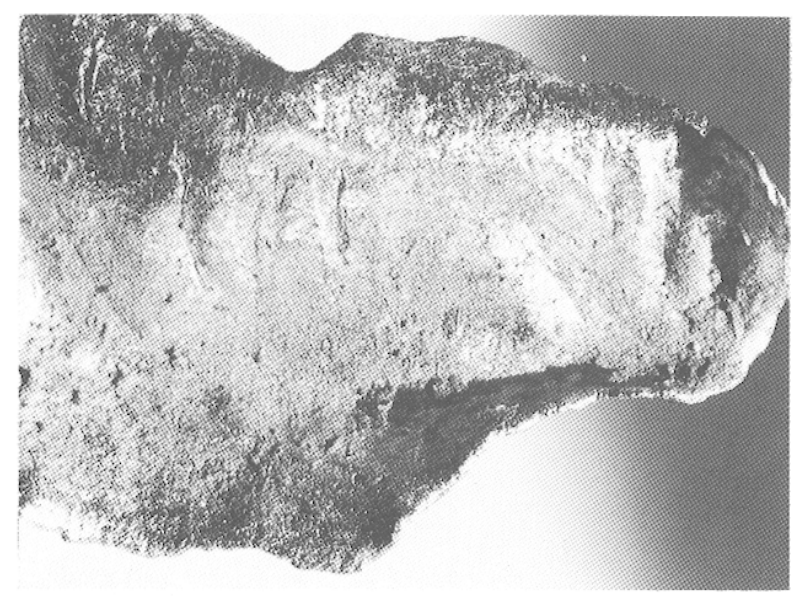

Fig. 16 - Casque d'Agris. Face B du fragment. Détail.

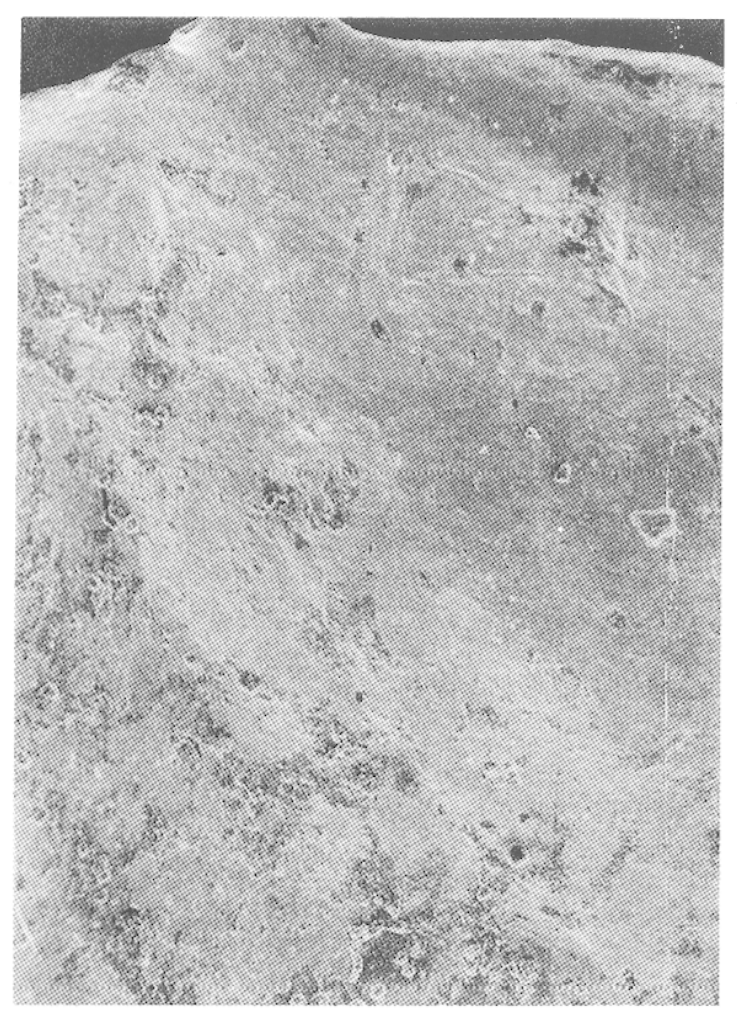

Fig. 15. - Casque d'Agris. Face A du fragment. Détail.

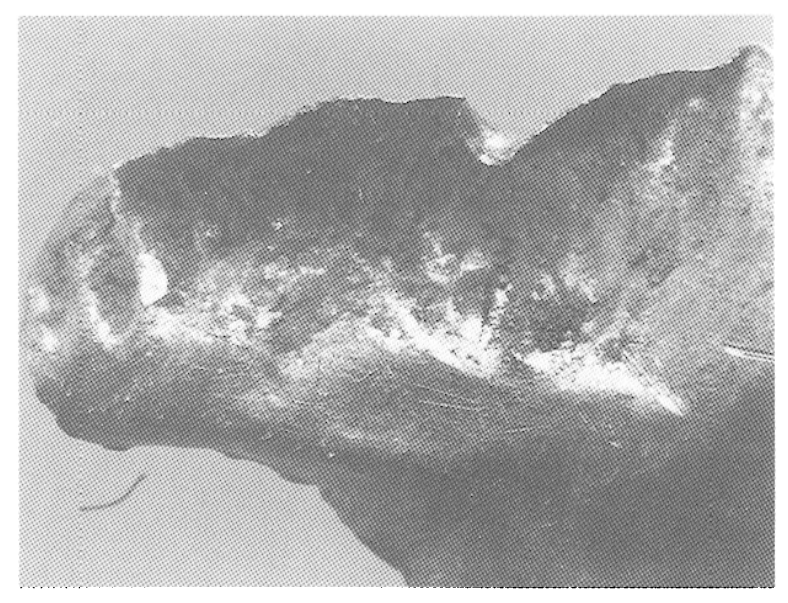

FACE A, MILIEU DU BORD SUPÉRIEUR

I a surface est très lisse, particulièrement audessus de la diagonale entre les angles supérieur gauche et inférieur droit. On remarque au-dessous de cette diagonale une série de dépressions accidentées de contour très irrégulier qui apparaissent en taches sombres. Elles pourraient être dues à des adhérences de la peau lors de la frappe. Les impacts sous forme de petits cratères sont peu nombreux. On note le bord festonné d'une nappe, coïncidant à peu près avec la diagonale, qui s'avance vers l'angle supérieur droit. Une autre nappe est moins visible en haut à droite. Le lissage est de très bonne qualité, sans doute effectué à l'aide d'une peau, sans rapport avec le lissage sommaire du fragment d'Amfreville. Quelques rayures modernes en triangle vers le haut de l'image.

Fig. 17 - Casque d'Agris. Face A, milieu du bord supérieur. Photo MEB ENSMA $\times 50$ (octobre 1987). 


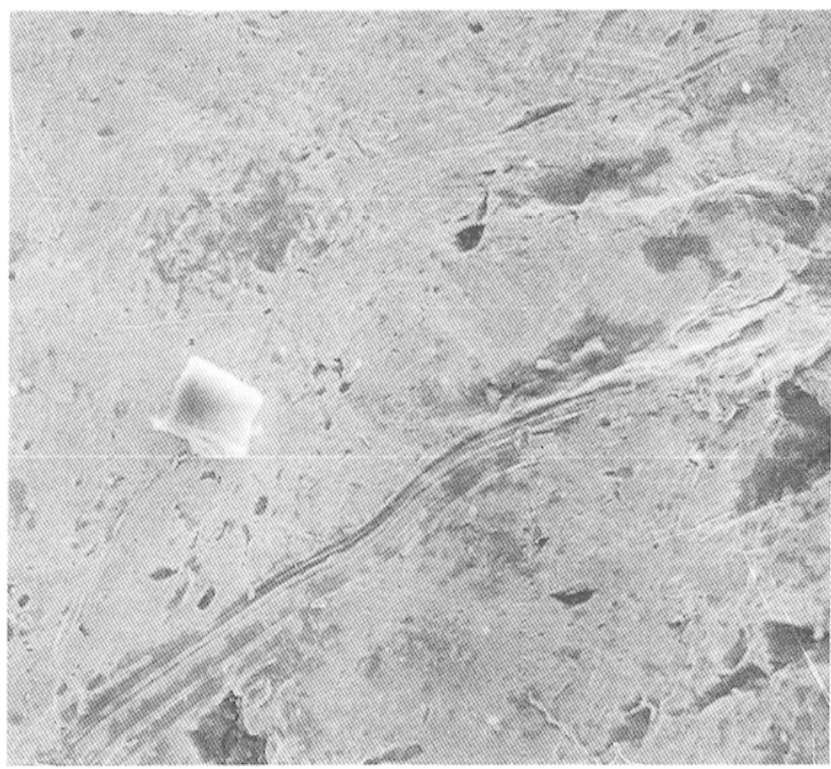

Fig. 18 - Casque d'Agris. Face A, centre. Photo MEB ENSMA $\times 300$ (octobre 1987).

\section{FACE A, CENTRE}

Une telle photographie rend parfaitement compte de la qualité remarquable du batteur d'or d'Agris. En effet le lissage à la peau a provoqué une surfacc très plane dans laquelle on a peine à discerner les sillons des nappes qui se dirigeaient vers le haut. Dans la moitié droite de l'image, on en distingue quatre au moins. Le plus important barre en diagonale-tout le champ jusqu'à l'angle inférieur gauche. On trouve quelques impacts çà et là. Le caractère parallèle de ces sillons et fronts de nappe pourrait faire croire à une frappe progressive classique $^{\mathbf{1 6}}$ si l'on ne possédait d'autres vues à des grandissements supérieurs.

16 Sur la frappe progressive, qui apparaît vers la fin du $\mathrm{II}^{\mathrm{e}}$ millénaire et $\mathrm{s}^{\prime}$ impose au $\mathrm{I}^{\mathrm{er}}$ dans le monde méditerranéen, cf. TOA, p. 80-83, pl. 1 a, c, $215 \mathrm{a}, 217 \mathrm{e}-\mathrm{j}$.

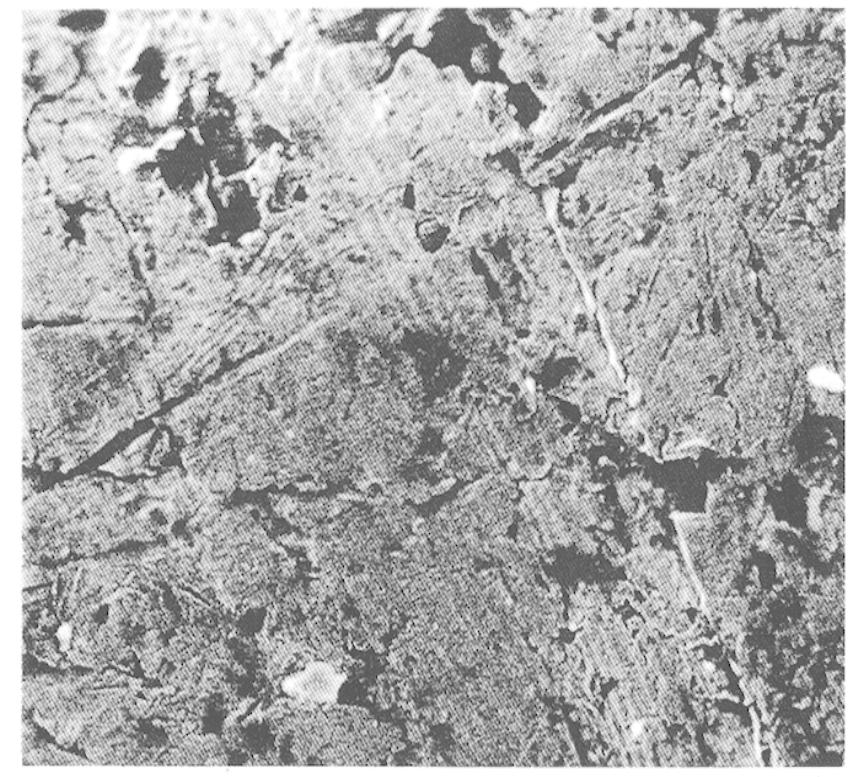

Fig. 19 - Casque d'Agris. Face $B$, centre.

\section{FACE $B$, CENTRE}

La qualité du travail est égale sur la face B. On observe la même surface plane qu'au grandissement $\times 300$. Le fait qu'il apparaisse des sillons de nappe entrecroisés, de deux directions différentes au moins, nous révèle une frappe verticale, exécutée avec le plus grand soin. On perçoit en effet un groupe de sillons parallèles orientés obliquement du haut en bas de la vue, un sillon sinueux de gauche à droite au tiers inférieur du champ (interrompu par des anfractuosités à droite), un front de nappe qui a retenu une impureté blanche dans l'angle inférieur gauche, des dépressions (noires) partiellement recouvertes par des lobes de nappes en haut à gauche. Des stries de lissage très légères sont perceptibles, dans une direction parallèle au premier groupe de sillons. 


\section{FACE B, VERS LE CENTRE}

L'intérêt de cette vue est de mettre en évidence les différences entre la frappe d'Amfreville et celle d'Agris à des grandissements similaires, $\times 1490 \mathrm{sur}$ la figure $8, \times 1510$ sur celle-ci. Sur la figure 8 , les sillons sinueux et contrariés restaient très apparents, révélant une frappe en tous sens et un lissage peu poussé. Ici, nous avons des sillons de nappe presque rectilignes bien visibles dans la partie droite, de deux directions principales. Le plus important aboutit dans l'angle inférieur droit. Il est recoupé par deux autres en croix de saint André, partiellement recouverts par le lissage dont on aperçoit les stries obliques de même direction que le premier sillon. Le lissage n'a pas réussi à niveler les quelques impacts (cratères) de frappe. On relève enfin quelques rayures modernes en bas et en haut à gauche. Quant à l'attaque chimique, très visible sur la figure 8 , elle est ici pratiquement inexistante comme on pouvait s'y attendre dans le cas d'un or presque pur.

\section{Position du problème}

Il apparaît donc que les feuilles de placage des casques d' $\Lambda$ mfreville et d'Agris ont été toutes deux élaborées par la méthode de la frappe verticale dite primitive suivie d'un lissage et appliquées par un brunissage sur le support de bronze. L'origine de la technique d'application de feuille d'or épaisse sur un support semble plus continentale ou hallstattienne que proprement méditerranéenne ${ }^{17}$. Néanmoins on perçoit des différences entre les deux frappes. La première est bien réalisée mais reste dans le cadre de la frappe primitive habituelle, mise en évidence par les reconstitutions (fig. 12 et 13). La frappe d'Agris est beaucoup mieux élaborée. Tout en appartenant à la même technique, elle semble annoncer la frappe progressive par sa régularité qui se traduit par des accidents parallèles. La finition est également bien supérieure, car le lissage à la peau a provoqué une surface beaucoup plus égale.

La signification de ces différences nous échappe pour l'instant car les études de la frappe de l'or à l'aide du MEB sont encore à leurs débuts. On doit

17 Cf. notes 9 et 10 . On comparera, entre autres, les épaisseurs considérables des feuilles d'appliques en or naturel de Hochdorf, notamment sur les cornes à boire, Trésors des princes celtes, catalogue d'exposition, Paris, RMN, 1987, p. 176, no: 82 à 90, fig. 231-232 (J. Biel) et analyses d'A. Hartmann p. 163, et du domaine laténien, de Schwarzenbach, P.-M. Duval, op. cit., p. 56-59, fig. 37-39, et d'Auvers-surOise, ibid., p. 64-65, fig. 49 (la technique du disque d'Auverssur-(Oise est particulièrement proche de celle des casques d'Amfreville et d'Agris).

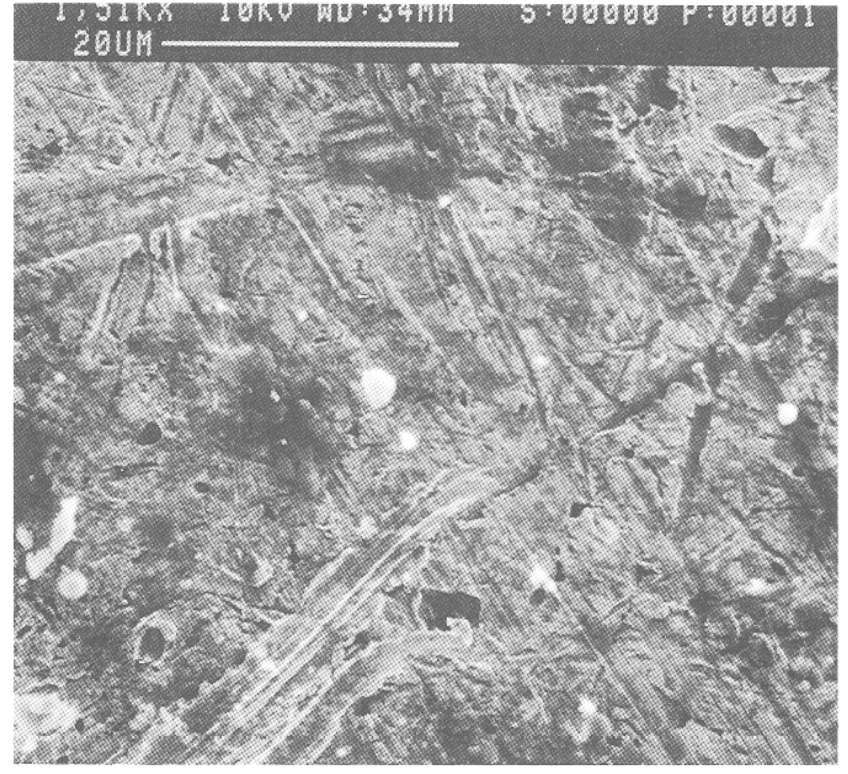

Fig. 20 - Casque d'Agris. Face B, vers le centre. Photo MEB ENSMA $\times 1510,10 \mathrm{KV}$, WD $34 \mathrm{~mm}$ (octobre 1987).

cependant reconnaître que les deux frappes gauloises révélées ici n'ont pas de rapport avec les frappes du domaine classique méditerranéen contemporain. Les placages du $\mathrm{IV}^{\mathrm{e}} \mathrm{s}$. en Grèce et même en Ibérie, où ils sont si nombreux, ne sont pas appliqués par les mêmes procédés et sont tous de frappe progressive ${ }^{18}$. En revanche, certaines feuilles ibériques, non plaquées, sont encore réalisées en frappe verticale ${ }^{19}$. Il serait donc intéressant de pratiquer un examen systématique au MEB des feuilles d'applique ou de placage, et même des feuilles d'or en général, des domaines du Hallstatt tardif (Hochdorf) et de La Tène, pour déterminer une géographie et une chronologie des méthodes de frappe, en corrélation avec des analyses d'or en micro-fluorescence $\mathrm{X}$ et à la microsonde. Cela permettrait d'envisager le problème de la provenance des ors ${ }^{20}$ et l'éventualité de centres de fabrication de feuilles et d'un commerce de feuilles d'or ${ }^{21}$, en tout cas de constater un accord ou un désaccord entre les influences stylistiques et les techniques. Ainsi, on pourrait mieux considérer le problème des lieux de fabrication de ces objets exceptionnels que sont les casques d'Amfreville et d'Agris.

\section{Gérard Nicolini}

18 Cf. note 16.

19 Cf. note 12.

20 A. IIartmann (cf. note 17) constate la parenté des ors de l'Allemagne du Sud et de Ifochdorf - peut-être fortuite avec les ors ibériques, alors qu'ils sont sans rapport avec les ors rhénans, souvent platinés.

21 Sur la spécialisation des ateliers, cf. $T O A$, p. 203. 


\section{L'UTILISATION DU MICROSCOPE ÉLECTRONIQUE À BALAYAGE DANS L'OBSERVATION DES OBJETS ANTIQUES}

Elle apporte des observations d'un type nouveau puisque cet appareil allie un grandissement important à une grande profondeur de champ et permet de donner sur les clichés une spectaculaire impression de relief que nous expliciterons plus loin. La réponse d'un objet à l'excitation par un faisceau d'électrons se traduit par plusieurs signaux enregistrés ou édités de diverses manières. Parmi les signaux produits à l'impact de la sonde, nous n'avons utilisé que les électrons secondaires ou de basse énergie donnant une image topographique et, quelquefois, les photons $\mathrm{X}$ des raies caractéristiques permettant d'accéder à la composition chimique de la zone irradiée; le microscope est alors réglé et utilisé comme microsonde. Le grandissement dépend de la tension d'accélération - en général dans cette étude, 10 kilovolts $(10 \mathrm{KV})$ - et de la distance de travail WD.

Sans entrer dans le détail de l'utilisation du MEB, il est intéressant de noter que les échantillons n'ont subi qu'une préparation réduite à un simple dépoussiérage, ce qui peut nuire à la qualité des photographies mais sauvegarde toute observation ou analyse ultérieure, avec le minimum de perte d'information par modification de l'objet étudié. Par objet étudié, on entend l'objet lui-même avec les incrustations, éventuellement dues à la fabrication ou au montage d'origine, et les traces des agressions chimiques et mécaniques qu'il a subies au cours du temps. Dans le cas où l'on avait besoin d'incliner l'objet pour une bonne observation, on a utilisé, pour le fixer sur le porte-échantillon, une petite goutte de colle à l'argent (soluble dans l'acétone) déposée sur une extrémité. Mais le plus souvent, l'objet a simplement été posé sur le porte-échantillon adéquat. On a aussi limité la tension d'accélération des électrons, d'une part afin d'éviter le marquage par irradiation, d'autre part en raison de l'existence de zones peu conductrices. On a aussi limité l'intensité du faisceau et on s'est gardé d'irradier longuement une même surface.

Revenons sur l'impression de relief donnée par le MEB. Le MEB donne de l'échantillon une image en perspective cavalière au lieu de la perspective conique perçue dans la vision binoculaire. Sur l'image, le sens du relief est donné par la localisation des ombres par rapport à la position du détecteur, autrement dit le sens du relief est fonction de la position du détecteur. Seule, l'observation de paires d'images stéréographiques rétablit la perspective conique. Dans le cadre forcément restreint de cet article, nous n'avons pas jugé nécessaire la publication de telles photographies.

Les manipulations auxquelles ont été livrés les échantillons peuvent être qualifiées de micro-investigations. Rappelons que l'image, traduction du spécimen examiné, peut être plus ou moins fidèle à la réalité. Les diverses images correspondent à autant d'objets qui sont chacun une certaine sélection effectuée sur l'objet afin de progresser dans la caractérisation de celui-ci ${ }^{22}$.

Le microscope électronique à balayage apporte certaines informations nouvelles. Nous nous sommes efforcés de prendre des plages caractéristiques et représentatives, mais les images produites ici ne représentent que d'infimes parties des petits objets examinés.

\section{Bernard Bouchet}

22 J. Philinfre, Microanalyse et microscopie électronique à balayage, Les Ulis, Les éditions de physique, 1979. 